\title{
Totalität und Vielfalt - gesellschaftliche Wirklichkeit als multidimensionaler Zusammenhang
}

Die Marx'sche Kritik der politischen Ökonomie bietet für drängende Fragen unserer Zeit wichtige Analyseinstrumente. Mit der Rekonstruktion der Strukturen der kapitalistischen Produktionsweise bringt Marx eine Dimension des Sozialen in den Blick, die globale Abhängigkeiten in Form gewaltsamer und krisenhafter Zwänge hervorbringt. Marx erfasst die kapitalistische Produktionsweise als einen historischen Strukturzusammenhang, der unserem In-der-Welt-Sein bestimmte Formen und Dynamiken vorgibt und spezifische Hierarchisierungen sowie versachlichte Herrschaftsverhältnisse hervorbringt. Insofern bietet er wichtige Instrumente der Gesellschaftskritik an, denn wenn diese Strukturen in ihrer historischen Gewordenheit erfasst werden, können sie auch als Gegenstand menschlicher Gestaltungskompetenz erscheinen.

Die vielen Diskussionen um die Frage, inwiefern oder ob überhaupt gesellschaftliche Phänomene ursächlich auf diese kapitalistischen Strukturen zurückzuführen sind, haben andererseits deutlich gemacht, dass man mit der Kapitalismusanalyse noch keine Gesellschaftsanalyse geleistet hat. Die Marx'sche begrifflich-theoretische Rekonstruktion der kapitalistischen Produktionsweise kann nicht den Anspruch erheben, alle Phänomene der empirischen Wirklichkeit umfassend zu erklären. Fraglich erscheint generell, inwiefern einzelne Phänomene und Ereignisse in irgendeiner Weise kausal auf übergreifende Strukturzusammenhänge zurückgeführt werden können. Insbesondere poststrukturalistische, postmarxistische und queer/feministische Ansätze heben Vielfältigkeit, Differenzen und Instabilität von Ordnungen hervor. Allerdings wird im Rahmen dieser Debatten auch immer wieder diskutiert, inwiefern diese Dimensionen in einem inneren Zusammenhang mit der kapitalistischen Verfasstheit der Gesellschaftsform gesehen werden müssen. So weist beispielsweise Antke Engel darauf hin, dass „eine Destabilisierung von Zwei-Geschlechter-Ordnung und normativer Heterosexualität oder ein Feiern geschlechtlicher und sexueller Ambiguitäten [...] nicht automatisch mit einem Abbau von Dominanz- und Ungleichheitsverhältnissen einher[geht]“ (Engel 2002: 22). Engel stellt damit die Frage nach gesellschaftlichen Bedingungen von Pluralisierung und Individualisierung und 
begreift diese als Bestandteile und Effekte „spätmoderner gesellschaftlicher und ökonomischer Entwicklungen“ (Engel 2002: 194).

Gerade vor diesem Hintergrund lohnt sich eine Re-Lektüre der Marx’schen Texte, die ihren Fokus auf deren Gegenstand und Geltungsanspruch legt. Marx analysiert die kapitalistische Produktionsweise als Strukturzusammenhang und argumentiert dabei auf einer Analyseebene, die so abstrakt ist, dass sie, für sich betrachtet, keine Aussagen über konkrete historische Gesellschaftsformationen zulässt. In diesem Sinne ist die Strukturanalyse der Marx’schen Kritik der politischen Ökonomie als begrifflich-theoretische Rekonstruktion einer Dimension des Sozialen zu verstehen und nicht als eine substanzielle Theorie über eine konkrete Gesellschaft (Althusser 1972: 59ff): „Gegenstand der Marxschen Analyse im 'Kapital' ist im strengen Sinne nicht der empirisch vorfindbare Kapitalismus des 19. Jahrhunderts, sondern ein durch Abstraktion davon gewonnener idealisierter Gegenstandsbereich“ (Eberle 1981: 174).

Eine solche Klärung des Gegenstands der Marx’schen Analyse ermöglicht produktive Anschlüsse, die sich nicht in festgefahrenen Gegenüberstellungen von System/Stabilität und Vielfältigkeit/Instabilität oder großen Erzählungen und kleinen Geschichten erschöpfen. Mit der Marxschen Analyse lassen sich in der kapitalistischen Produktionsweise begründete strukturelle Dynamiken benennen, die dazu führen, dass es durch Pluralisierung von Differenzen und Bedeutungen hindurch immer wieder systematisch zu Herrschaftssicherung und zur Reproduktion hierarchischer Verhältnisse kommt. Zugleich ist diese Analyse insofern unzureichend, als sie die Vielfältigkeit der Regulierungen und Materialisierungen dieser Macht- und Herrschaftsverhältnisse nicht erfassen kann. Wie ich hier am Beispiel Geschlechterverhältnisse deutlich machen möchte, ist es allerdings sinnvoll, dies nicht als eine Lücke der Marx'schen Analyse zu begreifen (die auf derselben analytischen Ebene zu schließen wäre), sondern vielmehr als eine Grenze, die auf die Notwendigkeit anderer analytischer Ebenen verweist.

Wie Dipesh Chakrabarty feststellt, besteht eine wichtige Herausforderung unserer Zeit darin, einen von beständiger Spannung durchzogenen Dialog zwischen gegensätzlichen Standpunkten aufrecht zu erhalten. Auf der einen Seite geht es darum, mit den Begriffen der Kapitalismusanalyse den Zusammenhang zu benennen, der in unserer historischen Gegenwart unsere Abhängigkeiten in versachlichten Gewaltverhältnissen strukturiert. Auf der anderen Seite gilt es aber die unterschiedlichen Weisen des Menschseins, die unhintergehbaren Differenzen in denen 'wir' unser In-der-Welt-Sein erleben, ernst zu nehmen (Chakrabarty 2000: 254). In diesem Sinne würde es nicht darum gehen, die Marx'sche Analyse durch weitere Dimensionen zu ergänzen oder gar zu vervollständigen. Vielmehr ginge es darum, diese Analyse in produktiver Spannung zu anderen Dimensionen und Analysen zu halten. 
Als paradigmatisches Feld für die Verhandlung der Frage des Gegenstands und Geltungsanspruchs der Marx'schen Analysen bieten sich die Geschlechterverhältnisse geradezu an. Schon in den Marx'schen Texten wird die konstitutive Verknüpfung von Produktion/Reproduktion und vergeschlechtlichter Arbeitsteilung immer wieder thematisiert. Im Anschluss an Martha Giminez gehe ich allerdings davon aus, dass das entscheidende analytische Angebot bei Marx nicht in dem zu finden ist, was er selber zum Verhältnis von Männern und Frauen und zur geschlechtlichen Arbeitsteilung geschrieben hat. Vielmehr sind seine Methode sowie seine Analyse der kapitalistischen Produktionsweise für feministische Gesellschaftskritik von Bedeutung (Giminez 2001: 68). Eine ähnliche Annahme findet sich in Ursula Beers feministischer Reinterpretation der Marx’schen Kapitalismusanalyse (Beer 1991a). Diese nehme ich hier zum Ausgangspunkt für die Diskussion, inwiefern die je historische Gestalt von sozialen Phänomenen, Subjekten und Praktiken über die Strukturen der kapitalistischen Produktionsweise hinaus auch in anderen konstitutiven Momenten nachvollzogen werden muss. Ich möchte deutlich machen, dass auf der analytischen Ebene, auf der Marx die Strukturen der kapitalistischen Produktionsweise rekonstruiert, beispielsweise keine Aussagen über Geschlechterverhältnisse oder Heteronormativität möglich sind. Wird dies als (notwendige) Grenze der Marx'schen Analyse gelesen, dann zeigen sich Anknüpfungspunkte an eine queer/feministische Perspektive, die ich im Anschluss an Judith Butler und Michel Foucault anhand der Dimensionen der symbolischen Ordnung sowie der Macht-Wissen-Regime skizziere. Wenn diese Dimensionen weder als Alternative noch als einfache Ergänzung betrachtet werden, sondern der Blick vielmehr auf die analytische Eigenständigkeit der unterschiedlichen Perspektiven gerichtet wird, eröffnen sich interessante Erkenntnismöglichkeiten. Gerade im häretischen Verhältnis (Balibar 2004) dieser drei Perspektiven lässt sich der paradoxe Zusammenhang von Totalität und Vielfältigkeit, der meines Erachtens kennzeichnend für die spezifische Dynamik moderner kapitalistischer Gesellschaften ist, erfassen.

\section{Ursula Beers feministische Marxinterpretation}

Ursula Beers durch Althusser geprägte Marxinterpretation erscheint für mein Anliegen interessant und produktiv. Beer differenziert begrifflich zwischen verborgenen Strukturen und sichtbarem Funktionszusammenhang, um analytische Abstraktionsebenen zu unterscheiden; die begrifflich-theoretische Rekonstruktion verborgener Strukturen dient als theoretischer Rahmen, um spezifische Bedingungen der Möglichkeit des sichtbaren Funktionszusammenhangs erfassen zu können. Beer greift damit Maurice Godeliers' Reformulierung der Marx'schen 
Unterscheidung von Wesen und Erscheinungsform auf, problematisiert aber zugleich deren objektivistischen Überhang. Eine materialistische Gesellschaftsanalyse müsse theoretisch klarer berücksichtigen, dass in den sichtbaren Artikulationen nicht nur die Funktionsbestimmung verborgener Strukturen eine konkrete Gestalt bekommt, sondern immer auch ein überschüssiges Moment wirksam ist - beispielsweise dass, was in der Form der Produktivkraft 'Individuum' „an Subjektivität brachliegt, nicht vernutzt wird, sich nicht vernutzen lässt“ (Beer 1991a: 138). Der Begriff der verborgenen Strukturen bezeichnet demnach eine Dimension gesellschaftlicher Wirklichkeit, die sinnlich-empirisch nicht unmittelbar erfassbar ist, sondern nur durch wissenschaftlich-theoretische Rekonstruktion zugänglich wird: die kapitalistische Produktionsweise. Diese Produktionsweise lässt sich als eine spezifische Kombination von Produktivkräften und Produktionsverhältnissen fassen, die auf der Existenz von Privateigentum an Produktionsmitteln beruht und als treibende Dynamik die Produktion von Mehrwert hervorbringt. Diese verborgenen Strukturen zeigen sich in der empirischen Wahrnehmung in einem sichtbaren Funktionszusammenhang. Damit sind konkrete Institutionen gemeint, Formen der Arbeitsteilung, gesetzliche Regelungen, Lebensformen, Praktiken. Dieser sichtbare Funktionszusammenhang ist in seiner materiellen Gestalt durch die verborgenen Strukturen konfiguriert, aber nicht determiniert. Das heißt, dass die konkreten Institutionen usw. in einem funktionalen Verhältnis zu den verborgenen Strukturen stehen, jedoch nicht einfach aus diesen Strukturen ableitbar sind: ,'Erscheinungsformen' wären nach dieser Auffassung nicht bloßer Ausdruck von sozialen Strukturierungsprinzipien, sondern immer zugleich auch Überschüssiges“ (Beer 1991a: 139).

Diese Unterscheidung, mit ihrer Implikation, dass der sichtbare Funktionszusammenhang zwar durch verborgene Strukturen bedingt ist, zugleich aber anderes - mehr - ist, als diese Strukturen, bietet einen Ansatzpunkt für meine These, dass es sinnvoll ist, die Kritik derpolitischen Ökonomie als begrifflich-theoretische Rekonstruktion einer spezifischen Dimension des Sozialen zu begreifen. Mit der Rekonstruktion des abstrakten verborgenen Strukturzusammenhangs ermöglicht Marx einen bestimmten analytischen Zugriff auf die Phänomene des sichtbaren Funktionszusammenhangs, der spezifische Formen erfassbar macht, durch die die gesellschaftliche Wirklichkeit konfiguriert wird. Marx fragt nun danach, wie diese Formen in ihrer spezifischen Gestalt erst in einem Strukturzusammenhang entstehen. Er verhandelt dies unter dem Begriff der trinitarischen Formel: Kapital, Arbeit und Boden erscheinen empirisch zunächst als unmittelbare, eigenständige Produktionsfaktoren, die jeweils ihren eigenen Anteil an der Produktion des gesellschaftlichen Reichtums haben (Marx 1989: 822f.). Die Kategorien 'Arbeit', 'Boden' und 'Kapital' scheinen auf eigenständige Einheiten zu verweisen, die jeweils die Quelle unterschiedlicher Formen von Einkommen sind (Lohn, 
Rente und Profit). Marx gibt sich allerdings nicht mit der Wahrnehmung dieser Phänomene im Sinne von vorgängigen 'Dingen' zufrieden, sondern rekonstruiert den gesellschaftlichen Zusammenhang, der diese Phänomene in ihrer spezifischen Formbestimmtheit und inneren Abhängigkeit voneinander überhaupt erst als 'Dinge' hervorbringt. Seine Analyse macht erkennbar, dass 'Arbeit' als Quelle von Einkommen eine bestimmte, historische Form der Verausgabung von Arbeitskraft gegen Lohn impliziert. Diese Form der Verausgabung von Arbeitskraft steht in konstitutivem Zusammenhang mit der historischen Form der Produktionsmittel als 'Kapital'. Beides ist wiederum ohne die Form des Privateigentums (an der eigenen Arbeitskraft oder an Produktionsmitteln als Kapital) nicht verständlich. Auch die einkommensgenerierende Potenz des 'Bodens' ist erst in ihrer historischen Form als Eigentum möglich, denn nur in dieser Form kann Boden einen Teil der erwirtschafteten Ressourcen als Einkommen an sich binden.

Durch die begrifflich-theoretische Ordnung des (verborgenen) strukturellen Zusammenhangs, der 'Arbeit', 'Kapital' und 'Boden' als Produktionsfaktoren hervorbringt, kann Marx argumentieren, dass die gesellschaftlichen Produktionsverhältnisse nicht eine Folge natürlicher Eigenschaften von Menschen und Dingen sind - oder eine bestimmte Organisation des Verhältnisses eigenständiger Faktoren. Seine Kritik der politischen Ökonomie setzt insofern auch nicht bei konkreten Problemen an, die sie als gegeben hinnimmt (z.B. ungleiche Verteilung knapper Ressourcen), um dann nach Lösungen für diese Probleme zu fragen (z.B. eine Umverteilung dieser Ressourcen). Vielmehr geht es dieser Kritik um die Strukturen, die diese Probleme als solche konstituieren. Es geht darum, die Frage zu stellen, in welchen Verhältnissen Dinge, Praktiken oder Beziehungen überhaupt in spezifischer Weise als Ressourcen hervorgebracht werden und in welcher Hinsicht sie knapp sind. Im Hinblick auf eine emanzipatorische Gestaltung kommt dadurch in den Blick, dass nicht nur konkrete Probleme bearbeitet werden können, sondern dass es vielmehr einer praktischen Reorganisation der Verhältnisse bedarf, die diese Probleme (immer wieder erneut, wenn auch möglicherweise in veränderten Figurationen des sichtbaren Funktionszusammenhangs) systematisch hervorbringen.

Mit einer solchen begrifflich-theoretischen Rekonstruktion kann jedoch nicht der Anspruch erhoben werden, alle Phänomene der empirischen Wirklichkeit umfassend zu erklären und so sind auf dieser Ebene beispielsweise auch keine Aussagen über Männer und Frauen oder über Geschlechterverhältnisse möglich. Hier setzt nun Beers Anliegen an, die Marx’sche Analyse des Kapitalismus begrifflich zu erweitern, um eine Analyse der Geschlechterverhältnisse zu ermöglichen. Sie sieht zwei Lücken in der Marx'schen Analyse. Zum einen kritisiert sie, dass der Begriff der Produktionsweise auf die marktorientierte Produktion beschränkt ist. Sie schließt daraus, „daß die historisch-materialistische Theorie in ihrer originären 
Gestalt nicht materialistisch genug ist, weil sie all das aus ihren Begriffen ausspart, was mit gesellschaftlicher Reproduktion unter- oder außerhalb des Produktionsprozesses von Waren zu tun hat" (Beer 1991b: 258). Beer insistiert darauf, dass die Tatsache, dass in jeder Gesellschaft nicht nur die materielle Produktion, sondern auch die biologische und kulturelle Re-Produktion der Menschen gewährleistet werden muss, keine Nebensächlichkeit ist, sondern durchaus ein wichtiges Thema der Gesellschaftstheorie. Biologische und kulturelle Reproduktion sind keine Begleiterscheinungen, keine automatischen Folgen der je spezifischen Produktion von Gütern und Diensten. Um diese gesellschaftstheoretische Relevanz der Reproduktion der Menschen begrifflich einzuholen, erweitert Beer das Konzept der Produktionsweise daher und unterscheidet zwischen einer 'Wirtschafts-' und einer 'Bevölkerungsweise'.

Die zweite Leerstelle der Marx'schen Analyse sieht Beer in der unzureichenden Bestimmung des Subjekts. Da sich die Kategorie der Arbeitskraft bei Marx aufdie abstrakte Verkörperung eines gesellschaftlichen Verhältnisses beziehe, existierten bei ihm keine Individuen aus Fleisch und Blut, sondern lediglich Träger sozialer Verhältnisse. Dem hält Beer entgegen, dass Subjekte nicht als abstrakte Formen agieren: „Wenn ich aber meine Arbeitskraft auf dem Markt anbiete, tue ich das nicht nur als potentielle Lohnarbeitskraft für einen Beschäftiger, sondern als Frau oder Mann“" (Beer 1991b: 260). Beer wirft also ein, dass eine Gesellschaftsanalyse berücksichtigen muss, „daß es ganz einfach auch Geschlechter geben muß, die ein solches Verhältnis konstituieren“" (Beer 1991b: 257).

Beer geht nicht davon aus, dass die bestehende geschlechtshierarchische Arbeitsteilung eine notwendige Erfordernis der kapitalistischen Produktionsweise ist, sondern will zeigen, inwiefern dieses Phänomen durch weitere Momente erklärt werden muss. Diese Momente sucht sie wiederum nicht in der natürlichen Gebärfähigkeit von Frauen, sondern führt die Tatsache, dass ausgerechnet Frauen den Großteil der nichtbezahlten Versorgungsarbeiten leisten, auf ein bereits vor dem Kapitalismus bestehendes patriarchales Prinzip zurück, ein „kulturelles Muster, das 'ganz selbstverständlich' Geschlechterherrschaft beinhaltete und voraussetzte" (Beer 1991a: 275). Beer geht also von einem Ineinandergreifen von patriarchalen Mustern und Produktionsweise aus, und untersucht dies anhand der Transformation der Geschlechterverhältnisse im Übergang von der ständischen zur bürgerlich-kapitalistischen Gesellschaft. Sie stellt fest, dass sich mit der Transformation zur kapitalistischen Moderne eine neue gesellschaftliche Organisation des Zusammenhangs von 'Wirtschafts'- und 'Bevölkerungsweise' durchsetzte und das dies wiederum mit einer neuen Form der Ungleichheit zwischen Männern und Frauen einherging.

Das kulturelle Muster der Geschlechterverhältnisse in der ständisch-agrarischen Gesellschaft bezeichnet Beer als Primärpatriarchalismus. Damit ist die 
Vorherrschaft des männlichen Familienoberhaupts gemeint, dessen Verfügungsgewalt über die Arbeitskräfte, sowohl der Ehefrau als auch des Gesindes, auf Eigentum an Grund und Boden beruhte. In der ständischen Gesellschaft bildete der Wirtschafts- und Familienverband die räumliche und funktionale Einheit der Organisation von Produktion und Generativität. Das Recht auf EheschlieBung (und damit auf legitime Familiengründung) war an den Nachweis einer ausreichenden Existenzsicherung gebunden. Besitzlose, die die Mehrheit der Bevölkerung ausmachten, waren in der ständischen Gesellschaft also nicht ehefähig. Beer interpretiert die entsprechenden gesetzlichen Regelungen im Familien- und Arbeitsrecht als sichtbaren Ausdruck der spezifischen Vergesellschaftungsmodi des ständischen Patriarchalismus; sie dienten der Aufrechterhaltung von Geschlechterungleichheit und Eigentumsordnung (Beer 1991a: 164).

Mit der Transformation zur kapitalistischen Produktionsweise veränderte sich die Vergesellschaftung von Arbeit und Generativität. Die kapitalistische Produktionsweise brachte die Verallgemeinerung der Lohnarbeit mit sich, wodurch neue Möglichkeiten der individuellen Existenzsicherung entstanden, denn der Lebensunterhalt konnte nun, vermittelt über den Verkauf der Arbeitskraft gegen Lohn oder Gehalt, unabhängig von Besitz an Grund und Boden oder an Produktionsmitteln gewährleistet werden. Beer macht allerdings geltend, dass die Effekte dieser historischen Entwicklung nicht allein auf der Ebene der nun über den Markt vermittelten Produktion zu betrachten sind. Sie macht auf die gesellschaftstheoretische Bedeutung einer tiefgreifenden Reorganisation von Arbeit und Generativität aufmerksam, im Zuge derer die Erfordernisse der individuellen und generativen Reproduktion weitgehend in eine von der Erwerbsarbeit getrennte Privatsphäre verwiesen werden.

Beer führt diese Reorganisation auf eine neue strukturelle Problemlage zurück: „Eine (kapitalistische) Marktökonomie kann nur dann profitabel und rational arbeiten, wenn sie keinerlei Verantwortung für die gesellschaftlich-individuelle Reproduktion von Frauen und Männern zu übernehmen braucht, die über die Lohn- oder Gehaltszahlung hinausgeht" (Beer 1990: 24). Sie spricht hier auf der analytischen Ebene der verborgenen Strukturen eine spezifische Konstellation der kapitalistischen Produktionsweise an, die sich als Blindheit des Kapitals gegenüber den eigenen Existenzbedingungen charakterisieren lässt. Die lebendige Arbeitskraft wird zur Reproduktion des Kapitalverhältnisses gebraucht, die Sicherstellung der individuellen Reproduktion dieser Arbeitskraft wird jedoch weitgehend aus dem Kapitalverhältnis ausgelagert. Den empirischen oder sichtbaren Ausdruck dieser Veränderungen auf der Ebene der verborgenen Strukturen untersucht Beer am Beispiel der Transformation der Rechtssysteme. So wurden etwa die Einschränkungen der Ehefähigkeit aufgehoben und neue arbeits- und sozialrechtliche Bestimmungen eingeführt. Mit der Differenzie- 
rung der Wirtschafts- und Familieneinheit in zwei räumlich und funktional getrennte Institutionen geht die Ausdifferenzierung des Patriarchalismus zu einer Form einher, die Beer als doppelten Sekundärpatriarchalismus bezeichnet. Dieser beruht auf der Verfügung über Geldmittel und sichert den Männern eine zweifache Vormachtstellung, zum einen aufgrund ihrer privilegierten Stellung in der Erwerbsarbeit und zum anderen durch ihre Verfügung über die Arbeitskraft von Frauen im Privatbereich.

Indem Beer in ihrer Untersuchung den Wandel der sozialen Ungleichheit der Geschlechter zum Wandel der Produktionsweise in Bezug setzt, kann sie strukturelle Rahmenbedingungen erfassen, die wesentliche Bedingungen der Möglichkeit der konkreten Gestalt der Geschlechterverhältnisse konstituieren. Im Mittelpunkt steht dabei die strukturelle Auslagerung wichtiger Aufgaben der individuellen Reproduktion in den 'Privatbereich'. Auf der abstrakten Ebene des verborgenen Strukturzusammenhangs lässt sich die kapitalistische Produktionsweise somit als Verwertungsprozess beschreiben, bei dem die Mehrwertproduktion als Relevanzkriterium für den Einsatz von Produktivkraft auftritt. Da sich der Bereich der individuellen und generativen Reproduktion in weiten Teilen nicht als Sphäre rentabler Kapitalanlage eignet, wird er der 'privaten' Verantwortung überlassen. Es entsteht eine strukturelle Notlage in Gestalt eines gesellschaftlichen Widerspruchs zwischen dem Verwertungsinteresse der Einzelkapitale und deren Gleichgültigkeit gegenüber den eigenen Existenzbedingungen. Hier greift der (patriarchal geprägte) Staat mit sozialpolitischen Bestimmungen und Maßnahmen regulierend ein. Beer zeigt in ihrer Untersuchung, wie tradierte kulturelle Muster der Geschlechterungleichheit in der Transformation der Produktionsweise wirksam werden und in die konkrete materielle Gestalt der Vergesellschaftung von Arbeit und Generativität eingelassen sind. Sie betrachtet die Transformation zum Sekundärpatriarchalismus moderner kapitalistischer Gesellschaften als „historisch-spezifische Antwort“ auf das Problem, wie eine industrielle Gesellschaft ihre „biologisch-natürliche Bestandssicherung“ (Beer 1993: 20) organisiert.

\section{Queer/feministische Problematisierungen}

Beers Vorschlag einer an strukturalistische Lesarten anknüpfenden feministischen Interpretation der Marx'schen Analyse mit ihrer Unterscheidung von verborgenem Strukturzusammenhang und empirisch wahrnehmbarer Realität ist für die Frage, wie unterschiedliche Macht- und Herrschaftsverhältnisse in ihrer kapitalismusspezifischen Konfiguration zu analysieren sind, sehr produktiv. Allerdings würde ich Beers Strategie einer kritischen Erweiterung der Marx’schen Analyse nicht ohne weiteres folgen. Anhand einer queer/feministischen Proble- 
matisierung der beiden Leerstellen, die sie bei Marx ausmacht, soll im Folgenden deutlich werden, inwiefern es sich hier nicht um theoretische Lücken handelt, die durch substanzielle Annahmen über Realsubjekte oder biologisch-natürliche Bestandserhaltung gefüllt werden können. Das, was Beer als Lücken ausmacht nämlich die Geschlechtlichkeit der Subjekte sowie die Frage der Reproduktion der Menschen - sind vielmehr als Grenzen der Marx'schen Analyse zu begreifen. Diese Grenzen verweisen auf weitere Analysedimensionen.

Ich beginne mit dem Begriff des geschlechtlichen Realsubjekts, über den Beer das Phänomen der hierarchischen Geschlechterverhältnisse in die Analyse kapitalistischer Gesellschaften einbinden will. Gegen Beers Annahme, dass es in allen bisherigen Gesellschaften neben der Produktion von Lebensmitteln auch die Produktion des Lebens selbst, die Erzeugung von neuen Menschen gegeben hat, ist grundsätzlich nichts einzuwenden. Auch ihre weiterführende Annahme, dass diese Reproduktion der Menschen in jeweils spezifischen Formen organisiert ist und dass die soziale Konstruktion von Geschlechtern eng mit diesen sozialen Formen verknüpft ist, ist durchaus sinnvoll. Beer führt den Begriff des (geschlechtlichen) 'Realsubjekts' jedoch ein, ohne dessen Genealogie systematisch in die Analyse einzubeziehen und setzt dadurch eine vorgängige Existenz von Männern und Frauen voraus, die in einer körperlichen, auf Fortpflanzung bezogenen Zweigeschlechtlichkeit begründet ist. Sie nimmt auf diese Weise etwas gesellschaftstheoretisch höchst Voraussetzungsvolles, nämlich die Zweiteilung der Menschheit in Männer und Frauen, als gegebenen Ausgangspunkt für Gesellschaftstheorie. Diese unhinterfragte Bezugnahme auf ein 'Realsubjekt' führt dann wiederum dazu, dass gesellschaftliche Akteure bei Beer vor allem als 'Männer' und 'Frauen' erscheinen und soziale Kämpfe als Bestrebungen männlicher Dominanzerhaltung im Rahmen neuer Produktionsverhältnisse thematisiert werden. Beides bleibt dabei unterbestimmt, denn Differenzen unter Frauen (und unter Männern) sowie damit verbundene unterschiedliche und teilweise konträre Interessenlagen können auf diese Weise nicht analytisch gleichrangig erfasst werden. ${ }^{1}$ Beers Annahme, dass die Lohnarbeitskraft nur als geschlechtliches Wesen denkbar sei, unterläuft letztlich ihre eigene Annahme, dass der Kapitalismus nicht notwendiger Weise auf hierarchische Geschlechterverhältnisse angewiesen ist. Mit ihrer Prämisse, dass Subjekte Männer oder Frauen sind und dem damit verbundenen Blick auf männliche Herrschaft (oder Patriarchat) gerät tendenziell die enorme

1 Frauen sind keine homogene Gruppe (Männer im Übrigen ebenso wenig) und Aussagen über Frauen, die nicht zumindest kontextualisiert und in ihrem Geltungsanspruch eingegrenzt werden (von welchen Frauen ist die Rede?), führen dazu, dass bestimmte Lebenssituationen, Probleme und Bedürfnisse (zumeist die der weißen Frauen der Mittelschicht in den USA und West-Europa) verallgemeinert werden. 
Vielfältigkeit und Flexibilität von Machtverhältnissen und Hierarchisierungen in kapitalistischen Gesellschaften aus dem Blick. Empirisch hat sich längst erwiesen, „dass das als Ware 'Arbeitskraft' agierende Subjekt durchaus ein weibliches Individuum sein darf - sofern es in entsprechender Weise von 'Sozialballast' frei ist" (Nickel 2002: 5). Dies wirft wiederum die Frage auf, wie - mit wessen Hilfe, mit welchen Mitteln, auf wessen Kosten - diese weiblichen Individuen sich von ihrem 'Sozialballast' befreien und inwiefern damit wiederum andere (zum Beispiel rassifizierte) Figurationen von 'Realsubjekten' verbunden sind. ${ }^{2}$

Eine Genealogie des Realsubjekts erfordert den Blick auf weitere verborgene Strukturzusammenhänge. Im Anschluss an Judith Butler lässt sich beispielsweise die Ordnung der Zweigeschlechtlichkeit als eine wichtige strukturelle Dimension der Gesellschaftsanalyse benennen. Mit dem Begriff der heterosexuellen Matrix erfasst Butler eine spezifische Figuration der symbolischen Ordnung, die die Geschlechtszugehörigkeit zu einer Bedingung der sozialen Lebensfähigkeit von Subjekten werden lässt. Subjekte müssen sich im Rahmen dieser Ordnung entweder als Mann oder als Frau zu erkennen geben und als solche erkennbar sein, um als normale und kompetente Subjekte anerkannt zu werden. Butler bezeichnet die binäre Geschlechterordnung als eine historische Bedingung der Intelligibilität, die Individuen überhaupt als Subjekte lebensfähig macht.

Wichtig dabei ist, dass die Geschlechtsidentität für Butler nicht in einer inneren Substanz und Körperlichkeit des Individuums begründet ist. Vielmehr begreift sie die Wahrnehmung von Körpern in einem binären Muster als männliche und weibliche Geschlechtsköper als eine Hervorbringung der heterosexuellen Matrix (Butler 1991). Diese Matrix stellt einen zirkulären Verweisungszusammenhang zwischen körperlichem Geschlecht (sex), sozialem Geschlecht (gender) und Begehren (desire) her und naturalisiert diese Kopplung: Das (sexuelle) Begehren richtet sich - als heterosexuelles Begehren - auf das jeweils andere Geschlecht und verweist daher auf das soziale Geschlecht, das wiederum an das körperliche Geschlecht anknüpft. Die vermeintliche Naturgegebenheit dieser Verbindung stabilisiert sich durch eine weitere Verknüpfung, nämlich der von Begehren, Sexualität und Fortpflanzung, die eine zirkuläre Evidenz hervorbringt: „Frauen bekommen Kinder, dafür brauchen sie Männer. Und weil dies so ist, lieben

2 In aktuellen Diskussionen um die Auslagerung 'individueller' Reproduktionsarbeiten an bezahlte Kräfte in europäischen Privathaushalten wird deutlich, dass derzeit nicht so sehr ein Verschwinden der Vergeschlechtlichung zu beobachten ist, es aber zu deutlichen Verschiebungen und Überlagerungen mit anderen Differenzen und Machtverhältnissen kommt (Gutiérrez-Rodríguez 2010). Hier finden Reorganisationen von Arbeitsteilung statt, die sich nicht sauber mit der Unterscheidung von Wirtschafts- und Bevölkerungsweise einfangen lassen und die auch nicht ausreichend durch das Muster einer vergeschlechtlichten Teilung der Arbeit zu erfassen sind. 
Frauen Männer und sind von Natur aus zur heterosexuellen Sexualität bestimmt. Diese Sexualität bedeutet Penetration, weil ja sonst keine Kinder zu zeugen sind. Männern geht es darum, Nachkommen zu haben. So ergänzen sich beide Geschlechter in ihrer polaren Opposition“ (Villa 2000: 145). Ähnlich wie Marx nicht einfach von einer eigenständigen Existenz der Kategorien Arbeit, Kapital und Boden ausgeht, gibt sich auch Butler nicht mit der Evidenz von sex, gender, und desire als vorgängige Tatsachen des menschlichen Lebens zufrieden, sondern fragt nach dem strukturellen Zusammenhang, innerhalb dessen vermeintlich eigenständige Momente - 'Geschlecht', 'Sexualität', 'Natur', 'sexuelle Praktiken' - ihre jeweils aufeinander verweisenden Bedeutungen erhalten. Die scheinbar natürliche Verknüpfung von körperlichem Geschlecht, sozialem Geschlecht und heterosexuellem Begehren lässt sich als ein machtdurchzogenes Diskursregime erfassen, das nicht nur die Intelligibilität der Subjekte strukturiert, sondern auch in gesellschaftliche Institutionen und soziales Handeln eingelassen ist.

Butler begreift die Geschlechtsidentität als eine performative Hervorbringung. Performative Sprechakte bringen Kraft der Bezeichnung soziale Realität hervor: „mit der ärztlichen Interpellation [...] wechselt das Kleinkind von einem 'es' zu einer 'sie' oder einem 'er'; und mit dieser Benennung wird das Mädchen 'mädchenhaft' gemacht, es gelangt durch die Anrufung des sozialen Geschlechts in den Bereich von Sprache und Verwandtschaft" (Butler 1997: 29). Diese 'ursprüngliche' Hervorbringung erzielt ihre dauerhafte Wirkung dann wiederum in Prozessen der ständigen, zitierenden Wiederholung: Das bei der Geburt zum Mädchen gemachte Kind wird „von den verschiedensten Autoritäten und über diverse Zeitabschnitte hinweg" (ebd.) immer wieder als solches angerufen und stellt sich selber als solches dar, wodurch der Effekt der Naturalisierung und Normierung immer wieder bestätigt und verstärkt wird. Die Worte, Gesten, Handlungen, mit denen diese performative Herstellung der Geschlechtsidentität immer wieder vollzogen wird, drücken keine vorgängige Geschlechtszugehörigkeit aus, erzeugen aber rückwirkend den Effekt eines „inneren Geschlechtskerns" (Butler 2001: 136).

Butler betrachtet die heterosexuelle Matrix allerdings nicht als eine geschlossene und universale Ordnung, sondern schreibt ihr vielmehr eine inhärente Instabilität und Veränderbarkeit zu, die sie darauf zurückführt, dass diese Ordnung nur in einer zitierenden Aufführung seitens der Subjekte besteht. Da jedes sprachliche Element also wiederholbar sein muss und in unterschiedlichen Kontexten, in verschiedenen Bedeutungsketten zitiert wird, unterliegt es in seiner Bedeutung immer Verschiebungen - und darin besteht die Möglichkeit der Umdeutung oder Resignifikation. So können etwa Mädchen ihr Mädchen-Sein in stark stereotypisierten Formen zum Ausdruck bringen oder es unkonventionell interpretieren und als Mädchen bestimmte Fähigkeiten für sich in Anspruch 
nehmen, die normativ als jungenhaft codiert sind. Auch können die anerkannten Konventionen der Interpretation des Mädchen-Seins in unterschiedlichen sozialen und kulturellen Kontexten variieren. Die Bedeutung von 'Mädchen' ist also nicht festgeschrieben; sie ist immer relational (in Bezug und in Abgrenzung zu der Bedeutung von 'Junge', 'Mann', 'Frau') und sie ist immer kontextualisiert.

Mit der Rekonstruktion der heterosexuellen Matrix als historischer Strukturbedingung der zweigeschlechtlichen Ordnung kann Butler eine Antwort auf die Frage geben, warum und inwiefern Subjekte Männer oder Frauen sind, ohne dies auf eine ahistorische oder natürliche Körperlichkeit zurückzuführen. Sie kann zugleich die prinzipielle Instabilität dieser Ordnung begründen und dadurch plausibel machen, dass sich die Geschlechterdifferenz immer in konkreten sozialen Kontexten im Zusammenhang mit anderen sozialen Strukturen materialisiert. Das von Beer in die Analyse eingebrachte 'Realsubjekt' wird auf diese Weise in weiteren Dimensionen historisiert: Es sind nicht einfach 'Männer' und 'Frauen', die als reale, körperliche Wesen gewissermaßen auf die historische Form der Arbeitskraft treffen - auch Männlichkeit und Weiblichkeit sind vielmehr ebenfalls als historische Formen zu betrachten.

Ähnlich wie das 'Realsubjekt' sind Beers Begriffe von Wirtschafts- und Bevölkerungsweise aus einer historisch-genealogischen Perspektive kritisch zu befragen. Wenn Beer die Transformation zum Sekundärpatriarchalismus moderner kapitalistischer Gesellschaften als „historisch-spezifische Antwort“ auf das Problem betrachtet, wie eine industrielle Gesellschaft ihre „biologisch-natürliche Bestandssicherung" (Beer 1993: 20) organisiert, dann ist dies aus queer/feministischer Perspektive insofern problematisch, als sie in ihrem Begriff der 'Bevölkerungsweise' Fortpflanzung, reproduktive Tätigkeiten (kulturelle Re-Produktion des Menschen) und Geschlecht weitgehend unvermittelt miteinander verknüpft. Generativität wird dadurch als Gattungsproblem thematisiert und mit naturalistischen und substanzlogischen Annahmen verbunden. Folgt man aber beispielsweise den Analysen Michel Foucaults zum modernen Dispositiv der Sexualität (Foucault 1983), dann lassen sich im Übergang zur kapitalistischen Moderne wichtige qualitative Veränderungen erkennen, die eine klare Unterscheidung von natürlichen Notwendigkeiten und kulturellen Mustern oder Antworten unsicher machen. ${ }^{3}$ Foucault wendet sich gegen die Annahme, Sexualität sei eine transzendentale natürliche - Essenz des Menschen, die in der abendländischen Moderne von den

3 Mit dem Begriff des Dispositivs bezeichnet Foucault einen Strukturzusammenhang, innerhalb dessen heterogene Momente des Sozialen (z.B. Diskurse, Institutionen, architektonische Formen der Raumorganisation, Gesetze) in einem Beziehungsgefüge stehen. Die Einheit eines Dispositivs kann über eine spezifische strategische Funktion im Sinne einer „Reihe von Absichten und Zielsetzungen“ (Foucault 1983: 116) rekonstruiert werden. 
prüden Vorstellungen christlich-bürgerlicher Sexualmoral machtvoll unterdrückt werde. Er geht vielmehr davon aus, dass gerade die vielfältige Thematisierung von Sexualität in der Moderne - in der Medizin, der Biologie, der Pädagogik, der Psychoanalyse, in religiösen und weltlichen Moralvorstellungen, in zwischenmenschlichen Beziehungen - überhaupt erst das Phänomen der Sexualität als spezifische Verknüpfung von körperlichen Funktionen, Begehren, Verhaltensweisen, Empfindungen, Moralvorstellungen und dergleichen hervorgebracht und zu einem besonderen Zugang zur Wahrheit des Menschen hat werden lassen.

Mit dem Konzept der Macht-Wissen-Regime stellt Foucault einen konstitutiven Zusammenhang zwischen spezifischen Formen, Institutionen und Praktiken der Wissensproduktion, Wahrheit und spezifischen Machtverhältnissen her. Seine historische Genealogie der Sexualität ist daher in eine Analyse der besonderen Machtkonstellation der Moderne eingebettet, die er als Bio-Macht bezeichnet und die sich insbesondere durch eine dezentrale Regulierung der Prozesse der Bevölkerung auszeichnet: Über Strategien der Gewaltenteilung, der Verwaltung, der Verteilung von Gütern und Diensten, der Erhebung von statistischen Regelmäßigkeiten, über Normierung und vorsorgende Risikoabwehr und vor allem auch über die normierte Selbstregulierung der einzelnen Subjekte wird eine Optimierung der Lebensprozesse der Bevölkerung angestrebt. Generativität erscheint mit der Durchsetzung der Bio-Macht als ein wichtiges, biopolitisch zu regulierendes Problem, denn in den Prozessen der historischen 'Entdeckung' der Bevölkerung als politisches und wissenschaftliches Problem bildeten Phänomene „wie das Verhältnis von Geburt- und Sterberaten, de[r] Geburtenzuwachs, die Fruchtbarkeit einer Bevölkerung usw.“ erste „Wissensobjekte“ und erste „Zielscheiben biopolitischer Kontrolle“ (Foucault 1999: 280f). Auch bei Foucault findet sich also eine analytische Stoßrichtung, die darauf zielt, Zusammenhänge zu rekonstruieren, innerhalb derer vermeintliche Evidenzen oder Notwendigkeiten in ihrer historischen Form erst hervorgebracht werden. Generativität kann auf diese Weise als ein spezifisches Dispositiv erfasst werden, das durch unterschiedliche Diskursstränge, Institutionen und Praktiken konstituiert ist: Medikalisierung der Fortpflanzung, Pädagogisierung des Umgangs mit jungen Menschen, sozialpolitische Regulierung durch Gesundheits-, Familien- und Bildungspolitik und auf Seiten der Individuen entsprechende Praktiken der verantwortungsvollen Selbstregulierung, etwa durch vernünftige Partnerwahl und Familienplanung sowie die Sorge um die richtige Erziehung und Förderung der Kinder.

Untersuchungen zur Entstehung und Entwicklung der bürgerlichen Familie untermauern die Annahme, dass die konkrete Bestimmung dessen, was unter 'Bevölkerungsweise' zu fassen sein könnte, höchst voraussetzungsvoll ist und keinesfalls aus vermeintlich der Generativität entspringenden Notwendigkeiten 
abgeleitet werden kann. ${ }^{4}$ Die Familie erfährt in der abendländischen Moderne einen Bestimmungs- und Funktionswandel, der in gewisser Weise als „Biologisierung der Definition von Familie“ (Klinger 2000: 32) aufgefasst werden kann: Statt der vormodernen Wirtschaftsgemeinschaft, die auch Gesinde und Dienerschaft umfasste, ist sie nun eine durch Blutsverwandtschaft gekennzeichnete und auf Liebe beruhende Verbindung, deren Aufgabe vor allem in der individuellen Reproduktion besteht. Sexuelles Begehren findet in dieser Anordnung in seiner normalisierten Form als Liebe Ausdruck; Elternschaft und Verwandtschaft sind in der Form der Familie heterosexuell normiert; Verwandtschaftsbeziehungen, vor allem aber die Beziehungen von Eltern und Kindern werden als Liebesbeziehungen erfahren (oder als solche idealisiert). Die Kleinfamilie, zur normalen Lebensform verallgemeinert, erscheint als Ort der unmittelbaren, persönlichen Beziehungen. Sie übernimmt wichtige Funktionen der individuellen körperlichen, aber auch geistig-emotionalen Reproduktion sowohl der Erwachsenen als auch der Kinder. Die Trennung von Haus- und Erwerbsarbeit ist also nicht eine historisch besondere Anordnung von weitgehend natürlichen Tätigkeiten. Statt das, was wir beispielsweise als wichtige Aufgaben der Pflege, Versorgung und Erziehung von Kindern betrachten, im Begriff der Bevölkerungsweise mit einer 'biologisch-natürlichen Bestandsicherung' kurzzuschließen, eröffnen Analysen im Anschluss an Foucault und Butler den Blick darauf, wie spezifische Selbstverständlichkeiten und Notwendigkeiten der Generativität in wissenschaftlichen Disziplinen, in der medialen Öffentlichkeit, aber auch in alltagsweltlichen Praktiken, in Familien, in Liebesbeziehungen performativ hervorgebracht, verhandelt und beständig verändert werden.

Von der Vielfalt und dem umkämpften Charakter dieser Phänomene abstrahiert die Marx'sche Analyse; sie erfasst nur bestimmte - für die kapitalistische Produktionsweise spezifische - Formen und Dynamiken. Eine wichtige gesellschaftskritische Leistung dieser Abstraktion besteht darin, dass sie einen bestimmten Imperativ erkennbar macht, dem Körperlichkeiten, Fähigkeiten und Bedürfnissen unterworfen sind, nämlich dem Imperativ der Verwertung. Dadurch kann sie wiederum konkreter bestimmte Dimensionen dessen erfassen, was Foucault als Ziel biopolitischer Regulierungen immer wieder anführt, ohne dafür eine historische Erklärung anzubieten: die Optimierung der Bevölke-

4 So setzte sich beispielsweise im Übergang zur Moderne die Vorstellung einer deutlich vom Erwachsenendasein zu unterscheidenden Lebensphase der Kindheit durch, wodurch überhaupt erst bestimmte sozialisatorische Praktiken entstehen konnten, etwa die besondere Pflege und vor allem pädagogische oder erzieherische und emotionale Zuwendung für junge Menschen. Wie der Blick auf die Praktiken und Technologien der Geburtenkontrolle, der Befruchtung und der Schwangerschaftsvorsorge deutlich macht, ist selbst die grundlegendste Bestimmung von Fortpflanzung als Zeugung, Austragen und Gebären nicht einfach eine biologische Tatsache. 
rungsprozesse im Sinne einer verwertungslogischen Effizienz- und Produktivitätssteigerung. Die vergeschlechtlichte Trennung von öffentlich und privat, von Erwerbsarbeit und Hausarbeit und die damit verbundene geschlechtliche Arbeitsteilung können als eine konkrete gesellschaftliche Lösung gesehen werden, die die Hervorbringung jenes „Geschichtsprodukt(s) [ermöglicht], das das Kapital allein nicht herstellt" (Negt/Kluge 1993: 23): die lebendige Arbeitskraft mit ihren spezifischen Fähigkeiten und Eigenschaften. Da in diese Lösung jedoch unterschiedliche Strukturzusammenhänge eingehen, die eine je eigene Dynamik haben, können diese sich auch unabhängig voneinander und gegeneinander verschieben. Denkbar ist also beispielsweise, dass die Geschlechterdifferenz an normativer Eindeutigkeit verliert oder es gar zu einem Verschwinden der binären Vergeschlechtlichung kommt. Dies allein würde die kapitalismusspezifischen Dynamiken und Hierarchien nicht beseitigen; vielmehr ist davon auszugehen, dass diese durch andere symbolisch-kulturelle Überformungen konfiguriert werden würden. Umgekehrt kann aber nicht davon ausgegangen werden, dass mit einer Veränderung der Produktionsweise zugleich auch (heteronormative und rassistische) Identitätszwänge und biopolitische Regulierungen verschwunden wären.

\section{Totalität und Vielfalt: Punkte der Häresie}

Während Butler und Foucault die beständige Umkämpftheit und Instabilität von Identitäten, Körperlichkeiten und Wahrheitsansprüchen fokussieren, verweisen die Analysen von Marx auf die deutungsunabhängige Systemstabilität als historische Besonderheit der kapitalistischen Produktionsweise. Wie ich eingangs, Chakrabarty zitierend, aufgeworfen habe, besteht eine Herausforderung an Gesellschaftstheorie und -kritik darin, beides in seinem paradoxen Zusammenhang zu betrachten und nicht gegeneinander auszuspielen. Auf diese Weise können spezifische Ungleichzeitigkeiten erfasst werden, die begreifbar machen, wie es trotz einer gleichbleibenden 'Anatomie' der Produktionsweise zur historischen Abfolge und synchronen Gleichzeitigkeit verschiedener Gesellschaftsformationen und Subjektivierungsweisen kommen kann, deren Vielfalt sich nicht auf ein einheitliches, entwicklungslogisches Prinzip zurückführen lässt. Um die prinzipielle Offenheit und Instabilität des Verhältnisses dieser verschiedenen Analysedimensionen zueinander zu fassen, erscheint mir Etienne Balibars Vorschlag, sie in ihren 'Punkten der Häresie' zu betrachten, sehr sinnvoll (Balibar 2004). ${ }^{5}$

5 Balibar verweist darauf, dass Foucault und Marx - obgleich sie in mehreren entscheidenden Punkten in Opposition zueinander stehen - im Hinblick auf die Frage der Transformation von Strukturen von Macht und Herrschaft aufeinander verwiesen werden können. 
Das Interessante daran ist, dass unterschiedliche Analysen auf diese Weise weder als Alternative noch als einfache Ergänzung betrachtet werden. Vielmehr wird der Blick auf deren analytische Eigenständigkeit gerichtet und diese insofern ernst genommen, als sie jeweils bestimmte Momente der Komplexität moderner Gesellschaften erfassen, die nicht durch eine der anderen Analysen eingefangen werden können (Meißner 2010: 255f.).

Mit seiner Analyse der kapitalistischen Produktionsweise kann Marx eine spezifische Versachlichung und Verselbständigung gesellschaftlicher Verhältnisse erkennbar machen. Er kann zeigen, inwiefern die Subjekte ihre gesellschaftlichen Verhältnisse als Wesenseigenschaften von Dingen und Menschen wahrnehmen (zum Beispiel als eigenständige Produktionsfaktoren - Arbeit, Kapital und Boden). 'Gesellschaff' erscheint dann wiederum als notwendige Regulierung dieser Natur von Dingen und Menschen. Marx bezeichnet dies als Verkehrung, da gesellschaftliche - also von den Menschen 'gemachte' - Verhältnisse gewissermaßen als Natur erscheinen. Zugleich macht er deutlich, dass die (wissenschaftliche) Erkenntnis dieses Zusammenhangs die wirkliche Gegebenheit dieser Verhältnisse nicht unmittelbar beeinflusst. Es handelt sich um einen Strukturzusammenhang, der "ohne Rekurs auf Deutungsprozesse handelnder Individuen erklärt werden“ kann (Eberle 1981: 211). Die von Marx visierte Versachlichung und Verselbständigung der gesellschaftlichen Dynamiken der Produktionsweise lässt sich durch diskursive Verschiebungen - durch Umdeutungen wie etwa eine moralische Aufwertung der unbezahlten Reproduktionsarbeit oder durch Flexibilisierung von Identitätszwängen - nicht verändern, vielmehr bedarf es einer praktischen Umgestaltung dieser Produktionsweise. Der kritische Impetus der Marx'schen Analyse besteht darin, diese vermeintlich sachlichen Verhältnisse in ihrer Gewordenheit deutlich zu machen und sie auf diese Weise zugleich als historische Bedingungen für kollektive Bearbeitung in den Blick zu bekommen:

„Der Kommunismus unterscheidet sich von allen bisherigen Bewegungen dadurch, daß er die Grundlage aller bisherigen Produktions- und Verkehrsverhältnisse umwälzt und alle naturwüchsigen Voraussetzungen zum ersten Mal mit Bewußtsein als Geschöpfe der bisherigen Menschen behandelt, ihrer Naturwüchsigkeit entkleidet und der Macht der vereinigten Individuen unterwirft." (Marx/Engels 1969: 70)

In diesem Zitat drückt sich die Hoffnung aus, dass die Menschen die soziale Bedingtheit ihrer gegenseitigen Abhängigkeiten erkennen und sich zusammenschließen können, um diese Verhältnisse im Sinne eines guten Lebens bewusst praktisch zu bearbeiten. Damit kann die Gestaltung und Reorganisation der Produktionsweise als konkreter Bezugspunkt für Bündnisse über Differenzen hinweg benannt werden. Zugleich kann dies wiederum nur ein sehr abstrakter Bezugspunkte sein, denn welche Macht die vereinigten Individuen auf ihre Produktions- und Verkehrsverhältnisse ausüben wollen können, welche Bedürfnisse 
sie haben, was sie sich unter einem guten Leben vorstellen, ist durch die Analyse der kapitalistischen Produktionsweise nicht zu klären. Die Analysen von Butler und Foucault geben wiederum wichtige Hinweise auf weitere Dimensionen, die gesellschaftstheoretisch in den Blick genommen werden sollten, um implizite Vorentscheidungen darüber, wer und was ein Mensch ist, zu vermeiden oder zumindest immer wieder als problematische Annahmen thematisieren zu können. In den Konflikten unserer Gegenwart geht es nicht allein um ökonomische Strukturen und Verteilungskämpfe, sondern auch darum, wie ein soziales Zusammenleben gestaltet werden sollte, welche Werte Geltung haben sollten, welche Selbstverhältnisse und Subjektivierungsweisen anzustreben sind.

\section{Literatur}

Althusser, Louis (1972): Einführung: Vom 'Kapital'zur Philosophie von Marx. In: Louis Althusser/ Etienne Balibar: Das Kapital lesen I. Reinbeck bei Hamburg, 11-93.

Balibar, Etienne (2004): Foucault-Marx, parallèles etparadoxes. In: Humanité 16.12.2004; http:// www.humanite.fr/2004-12-16_Cultures_-Foucault-Marx-paralleles-et-paradoxes (Zugriff am 4.5.2008).

Beer, Ursula (1990): Kapitalismus und Patriarchat. Die doppelte Vergesellschaftung von Frauen. In: Zeitschrift für sozialistische Politik, Heft 55, 23-25.

Beer, Ursula (1991a) Geschlecht Struktur Geschichte. Soziale Konstituierung des Geschlechterverbältnisses. Frankfurt a.M./New York (2. unveränd. Auflage).

Beer, Ursula (1991b): Zur politischen Ökonomie der Frauenarbeit. In: T. Brüsemeister u.a. (Hg.): Die versteinerten Verhältnisse zum Tanzen bringen. Beiträge zur marxistischen Theorie heute. Berlin, 255-263.

Beer, Ursula (1993): Geschlecht - Klasse - Struktur. In: Gabriella Hauch (Hg.): Geschlecht - Klasse Ethnizität. 28. Internationale Tagung der Historikerinnen und Historiker der Arbeiterbewegung. Wien/Zürich: Europaverlag, 14-26.

Butler, Judith (1991): Das Unbehagen der Geschlechter. Frankfurt a.M.

Butler, Judith (1997): Körper von Gewicht. Frankfurt a.M.

Butler, Judith (2001): Psyche der Macht. Das Subjekt der Unterwerfung. Frankfurt a.M.

Chakrabarty, Dipesh (2000): Provincializing Europe. Postcolonial Thought and Historical Difference, Princeton N.J.

Eberle, Friedrich (1981): Intentionales Handeln und gesellschaftliche Entwicklung. Frankfurt a.M.

Engel, Antke (2002): Wider die Eindeutigkeit. Sexualität und Geschlecht im Fokus queerer Politik der Repräsentation. Frankfurt a.M./New York: Campus.

Foucault, Michel (1983): Sexualität und Wahrheit. Bd. I: Der Wille zum Wissen. Frankfurt a.M.

Foucault, Michel (1999): In Verteidigung der Gesellschaft. Frankfurt a.M.

Giminez, Martha E. (2001): Le capitalisme et l'oppression des femmes: pour un retour à Marx. In: Actuel Marx 30/2001, 61-84.

Gutiérrez-Rodríguez, Encarnatión (2010): Migration, Domestic Work and Affect: A Decolonial Approach on Value and the Feminization of Labor. New York/London.

Klinger, Cornelia (2000): Die Ordnung der Geschlechter und die Ambivalenz der Moderne. In Sybille Becker, Gesine Kleinschmitt, Ilona Nord, Gury Schneider (Hg.): Das Geschlecht der Zukunft. Frauenemanzipation und Geschlechtervielfalt. Stuttgart, 29-63. 
Marx, Karl (1989): Das Kapital Bd. 3. MEW 25. Berlin.

Marx, Karl/Engels, Friedrich (1969): Die Deutsche Ideologie. I Feuerbach. MEW 3. Berlin, 13-77.

Meißner, Hanna (2010): Jenseits des autonomen Subjekts. Zur gesellschaftlichen Konstitution von

Handlungsfähigkeit im Anschluss an Butler, Foucault und Marx. Bielefeld.

Nickel, Hildegard Maria (2002): Marxismus und Feminismus - Facetten einer vielschichtigen Bezie-

hung.http//www.ucm.es/info/eurotheo/hismat/materiales/nickel.html (Zugriff am 19.11.03).

Negt, Oskar/Kluge, Alexander (1993): Geschichte und Eigensinn 1. Entstehung der industriellen

Disziplin aus Trennung und Enteignung. Frankfurt a.M.

Villa, Paula-Irene (2000): Sexy Bodies. Eine soziologische Reise durch den Geschlechtskörper. Opladen.

Niddastraße 64, 60329 FRANKFURT
Tel. (069) 67 9984
express-afp@online.de
www.express-afp.info

